\title{
Influence of intake parameters on combustion and emission performance of dual-fuel Marine diesel engines
}

\author{
Tao Dend ${ }^{1}$, Jialiang Huang, ${ }^{2}$, Deng $\mathrm{Hu}^{1}$, Jinyu $\mathrm{Fan}^{2}$, and Zixiao $\mathrm{Ye}^{3}$ \\ ${ }^{1}$ Marine Engineering Institute, Jimei University, Fujian, Xiamen 361021, China \\ ${ }^{2}$ Fujian Provincial Key Laboratory of Naval Architecture and Ocean Engineering, Fujian, Xiamen 361021, China \\ ${ }^{3}$ College of Navigation, Quanzhou Normal University, Fujian, Quanzhou, 362000, China
}

\begin{abstract}
We in order to study the influence of intake parameters on the combustion and emission of natural gas-diesel dual-fuel Marine engine, AVL FIRE simulation software was used to build a high pressure cycle model of combustion chamber based on 4190ZLC-2 Marine medium-speed diesel engine. The accuracy of the model was verified by comparing the simulation data with the cylinder pressure curve obtained from bench test. The influence of intake parameters on combustion and emission performance was studied by model simulation. The results show that the combustion quality can be improved by increasing the intake temperature, and the power performance and economy can be improved by appropriate intake temperature. Increasing the intake pressure has the effect of supercharging, appropriately increasing the intake pressure can obtain better power and emission, and can also avoid the rough operation of dual-fuel diesel engine and reduce the noise
\end{abstract}

\section{Introduce}

We in order to cope with the worsening global environment and the continuous shortage of fossil resources, the emission of Marine diesel engine has caused global environmental problems, so people turn to the gas-diesel dual-fuel engine [1]. Compared with traditional diesel engines, dual-fuel diesel engines have advantages such as lower emission pollution and better economy ${ }^{[2]}$. Gas-diesel dual fuel engines have been widely used on ships ${ }^{[3]}$. The combustion quality in the cylinder is closely related to the performance of the engine ${ }^{[4]}$, and the operating parameters have a great impact on the performance of the dual-fuel diesel engine [5]. German MAN Diesel \& Turbo in 2012 launched the ME - GI type natural Gas - Diesel dual fuel engine, the circulating high pressure direct injection Diesel technology, replacement rate is as high as $97 \%$, using EGR(Exhaust Gas Recirculation, Exhaust Gas Recirculation) can meet the IMO (International Maritime Organization, the International Maritime Organization) Tier III requirements. The dual-fuel diesel engine of Wartsila of Finland adopts low-pressure direct injection in cylinder and "micro-ignition" technology, which can realize full-load operation, with the replacement rate of natural gas reaching 95\%-99\% and HC emission greatly reduced ${ }^{[6]}$. Domestic pan jianfeng et al. ${ }^{[7]}$ Established a two-dimensional simulation calculation model by using FLUENT, and studied the influence of fuel injection duration and natural gas substitution rate on the combustion process of dual-fuel diesel engines. Wang limin et al. ${ }^{[8]}$ Studied the influence of different loads, natural gas substitution rate and excess air coefficient on combustion characteristics of dual-fuel engines by using test bench. Zhang ning et al. [9] Built a CFD Computational Fluid Dynamics three-dimensional jet model for dual-fuel diesel engines and studied the influence of EGR rate on the comprehensive performance of high-pressure direct jet engines. This paper starts with the main operating parameters of dual-fuel diesel engines, and adopts model simulation to study the combustion variation characteristics and pollutant generation rules in cylinder under different operating parameters, so as to provide a basis for the development of dual-fuel diesel engines.

\section{Establishment of research object and simulation model}

The research object of this paper is the 4190ZLC-2 ship 4-cylinder medium-speed exhaust turbocharged diesel engine produced by Jinan diesel engine factory. The main technical parameters are: cylinder diameter $\times$ stroke $190 \mathrm{~mm} \times 210 \mathrm{~mm}$, compression ratio $14: 1$, straight mouth ohm combustion chamber, nominal speed $1000 \mathrm{r} / \mathrm{min}$, nominal power $220 \mathrm{~kW}$, number of spray holes 8 , spray included Angle $150^{\circ}$.

According to the actual size of the combustion chamber of the original machine, the two-dimensional geometric model of the combustion chamber was first established by using auto-cad modeling software, and then imported into the ESE module of AVL_FIRE. As the ESE module will automatically generate three-dimensional moving mesh, and complete the grid division and inspection. This paper studies the combustion process in cylinder from inlet valve closing $\left(594^{\circ}\right)$ to exhaust valve opening $\left(841^{\circ}\right)$. In order to

*Corresponding author: jlhuang@jmu.edu.cn 
simplify the simulation calculation, as the combustion chamber of the original machine is symmetrical and the number of jet holes is 8 , one eighth of the combustion chamber is selected as the simulation experimental area to facilitate the intuitive reflection of combustion phenomenon, as shown in figure 1. In addition to selecting turbulent flow, droplet fragmentation, evaporation, combustion and emission submodels in AVL_FIRE, a chemical reaction mechanism file suitable for dual-fuel combustion should be added to the solver Settings.

Due to the limitation of test-bed condition, this article under the rated conditions in AVL_FIRE solver Settings in cylinder in the gas phase composition of the mass fraction of gas $(\mathrm{CH} 4)$ is 0 , through the model simulation of pure diesel engine cylinder pressure curve and the original machine frame of the measured cylinder pressure curve contrast, constantly adjust the relevant parameters, until the simulation cylinder pressure curve are essentially coincident with the original machine cylinder pressure curve. As shown in figure 2, the error within $5 \%$ is of high accuracy, which can be used for simulation research.

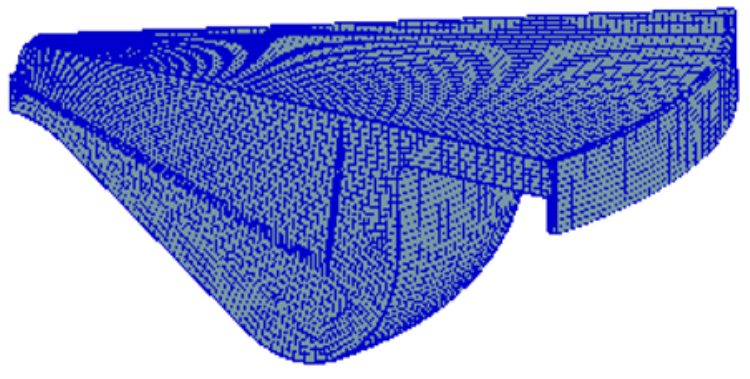

Fig. 1 1/8 combustion chamber $3 \mathrm{~d}$ mesh model

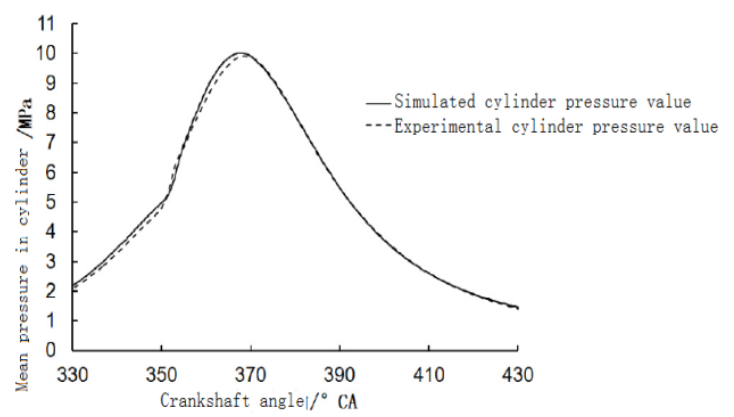

Fig. 2 comparison between simulation value and measured value of indicator diagram

\section{Analysis of influence of intake parameters on performance of dual fuel engine}

The performance of engine is closely related to combustion quality in cylinder. The performance of the engine can be optimized by properly organizing the combustion process in the cylinder. Intake parameters can affect the cylinder turbulence, material and energy transport, the combustion process will also have an impact on the performance of the engine. Here, inlet temperature $(325.15 \mathrm{k}, 335.15 \mathrm{k}, 345.15 \mathrm{k}, 355.15 \mathrm{k})$ and inlet pressure $(0.173 \mathrm{mpa}, 0.193 \mathrm{mpa}, 0.213 \mathrm{mpa}$,
$0.223 \mathrm{mpa}$ ) are selected to study the influence of these inlet parameters on the performance of dual-fuel diesel engines ${ }^{[10-11]}$.

\section{1 Analysis of influence of inlet temperature on performance of dual-fuel diesel engine}

Temperature has a great influence on chemical reaction, ignition delay and NO emission. As can be seen from figure 3 , with the increase of inlet temperature, the inflection point of temperature rise in the cylinder is advanced, the ignition time is advanced, the ignition delay period is shortened, and the maximum average pressure and average temperature in the cylinder are significantly increased. The higher inlet temperature accelerates the atomization and evaporation of oil and gas in the cylinder, intensifies the molecular collision movement, improves the combustion efficiency, makes the fuel burn fully, increases the heat release rate, and increases the average pressure and average temperature. It can be seen from figure 4 that when the inlet temperature increases, the indicating power tends to be flat with the increase of temperature, and the mass fraction of NO increases significantly. Higher inlet temperature speeds up the physico-chemical reaction rate in the cylinder, and the combustion process is completed. The heat release rate is large, and the increase of average pressure increases the indicating power ${ }^{[12]}$, and the increase of average temperature promotes the generation of NO.
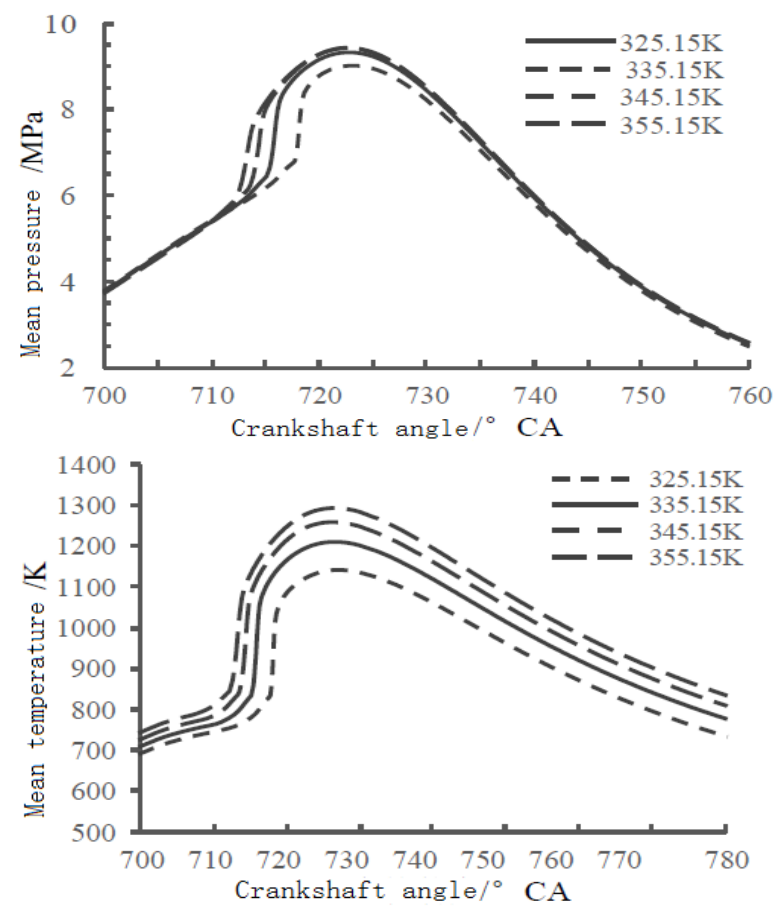

Fig. 3 influence of different inlet temperatures on cylinder pressure and temperature 

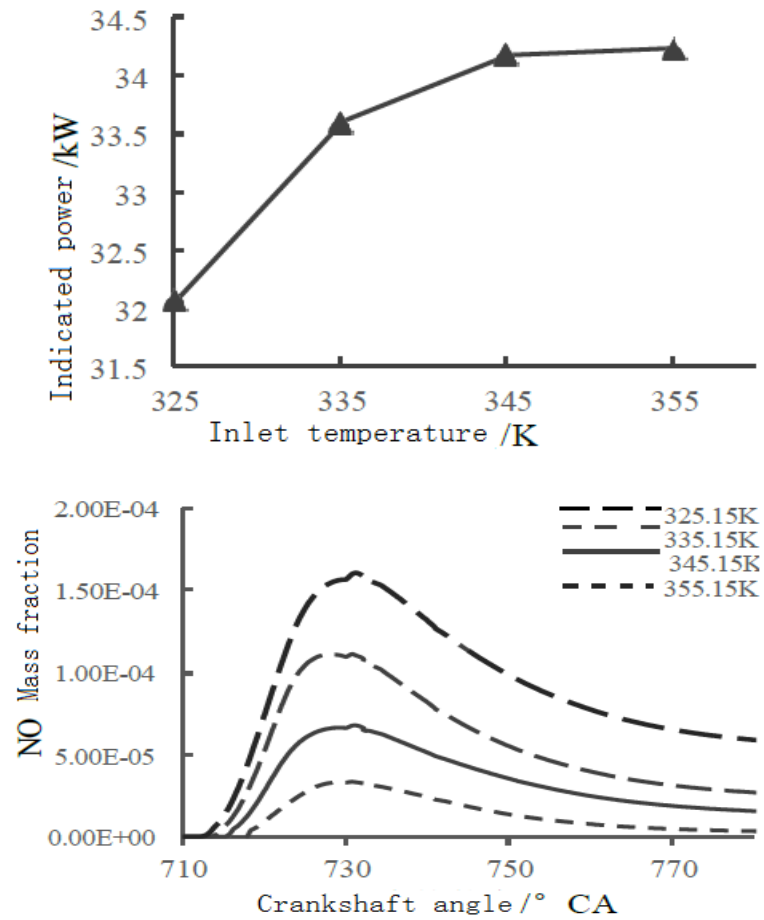

Fig. 4 influence of different inlet temperatures on indicating power and $\mathrm{NO}$ generation

\subsection{Analysis of influence of inlet pressure on performance of dual-fuel diesel engine}

Intake pressure has influence on turbulence, heat and mass transfer in cylinder. As can be seen from figure 5, with the increase of inlet pressure, the average pressure and peak value in the cylinder increase, which promotes the ignition time and inflection point of pressure rise to advance, and the average temperature in the cylinder decreases. When the intake pressure increases, the amount of gas in the cylinder increases, and the turbulent kinetic energy in the cylinder increases, which is beneficial to the mixed combustion of gas. However, if the intake pressure is too high, the highest burst pressure will be on the high side and the rough mechanical load of the diesel engine will be too large. Therefore, the intake pressure should be appropriately selected.

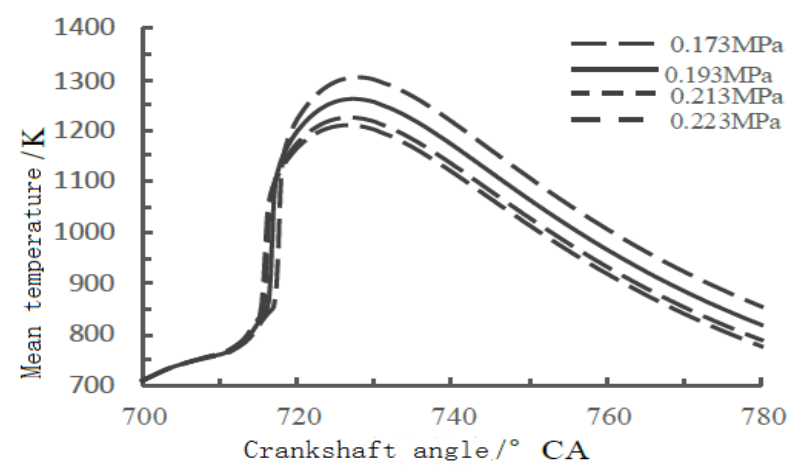

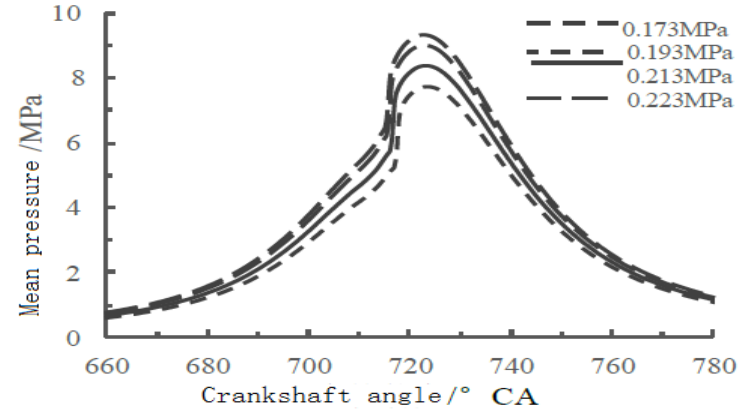

Fig. 5 influence of different inlet pressure on cylinder pressure and temperature

According to the analysis of figure 6, the higher the inlet pressure is, the lower the quality fraction of $\mathrm{NO}$ generation is, and the variation trend of $\mathrm{NO}$ generation is basically the same. FIG. 5 the increase of intake pressure will reduce the temperature in the cylinder, and the generation of $\mathrm{NO}$ is closely related to the high temperature, so the production of NO will also be reduced ${ }^{[13]}$. With the increase of air inlet pressure, the peak value of heat release rate and the ignition time are advanced. Due to the increase of air entering the cylinder, some heat will be absorbed in the cylinder. Meanwhile, the pressure of diesel fuel injection in the cylinder is relatively reduced.
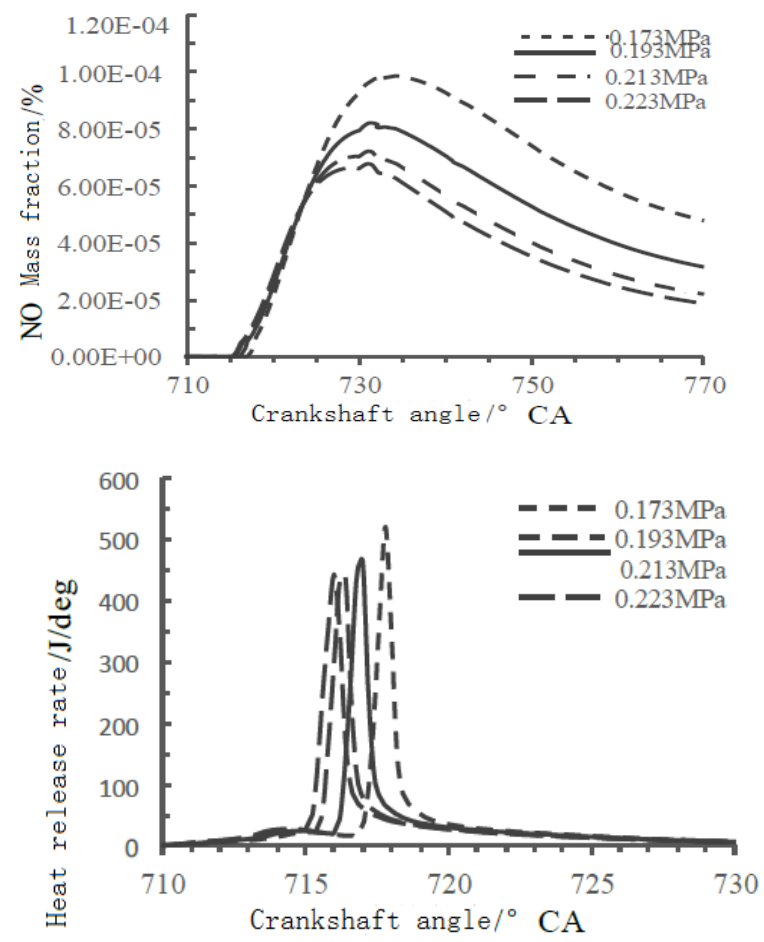

Fig. 6 effects of different inlet pressures on NO emission and heat release rate

\section{Conclusion}

In this paper, AVL-Fire software is used to establish a dual-fuel diesel engine simulation model by taking 4190ZLC-2 medium-speed Marine exhaust turbocharger diesel engine as the prototype. Moreover, the simulation results obtained through test data analysis are in good 
agreement with the test results, and the established model is relatively accurate. The influence of main operating parameters (natural gas substitution rate, inlet temperature, inlet pressure, injection advance Angle) on combustion and emission performance of dual-fuel diesel engine is studied by using the simulation model. The conclusions are as follows:

1) Temperature affects the physical and chemical reactions. The increase of intake temperature increases the average pressure, temperature and indicating power in the cylinder, and the NO emission increases. It is helpful to improve the performance and economy of dual fuel diesel engine by adopting proper intake temperature.

2) The increase of the intake pressure will effect of supercharging, the indicated power will increase, and the peak heat release rate, average temperature and NO emission in the cylinder will decrease. Proper increase of intake pressure can avoid rough operation and reduce noise of dual-fuel diesel engine, and obtain better power and emission.

\section{Acknowledgement}

Fund projects:

Fujian Natural Science Foundation funded projects(2017 J01486)

Fujian Natural Science Foundation funded Projects(2018J01499)

\section{References}

[1]. H. Aiguo. Internal Combustion Engines and Parts, 19(11) (2018).

[2]. W. Chao. Economic and Trade Practice, 21(6) (2018).

[3]. Q. Zhenqing. Petroleum Business Technology, 3(5) (2018).

[4]. H. Meng, W. Guoyang, L.Kang, et al.Ship Building of China, 56(2) (2015).

[5]. L. Weifeng, L. Zhongchang, W. Zhongshu, et al. Transactions of CSICE, 33(6) (2015).

[6]. H. Chaowei, N. Zhibin, Q. Bingjia, et al. Internal Combustion Engines and Parts, 17(31) (2018).

[7]. P. Jianfeng, T. Jiayu, H. Xiang, et al. Journal of Thermal Science and Technology, 17(5) (2018).

[8]. W. Limin, H. Yiyong, W. Zhigang, et al. Chinese Internal Combustion Engine Engineering,39(5) (2018).

[9]. Z. Ning, L. Jie, W. Junle. Transactions of CSICE, 36(6) (2018).

[10]. D. Lianchao. Study on combustion cycle variation of diesel/natural gas dual fuel engine. Changchun: Jilin University, (2015).

[11]. X. Zishun. Research on emission characteristics and control of natural gas engines. Jinan: Shandong University, (2017).

[12]. Z. Jia. Simulation study on the effect of biodiesel on diesel engine performance. Chengdu: Southwest
Jiaotong University, (2018).

[13]. Y. Hongliang, D. Shulin, S. Peiting, et al. Journal of Dalian Ocean University, 33(3) (2018). 